\title{
Oscillation criteria of certain fractional partial differential equations
}

\section{Di Xu' and Fanwei Meng ${ }^{1 *}$}

${ }^{*}$ Correspondence:

fwmeng@qfnu.edu.cn

'School of Mathematical Science, Qufu Normal University, Shandong, China

\begin{abstract}
In this article, we regard the generalized Riccati transformation and Riemann-Liouville fractional derivatives as the principal instrument. In the proof, we take advantage of the fractional derivatives technique with the addition of interval segmentation techniques, which enlarge the manners to demonstrate the sufficient conditions for oscillation criteria of certain fractional partial differential equations.
\end{abstract}

Keywords: Fractional partial differential equation; Oscillation; Riccati transformation

\section{Introduction}

The fractional calculus could be regarded as an age old but interesting theme. The appearance of the fractional differential equations is becoming more and more frequent. Kumar et al. [1,2] studied the fractional diabetes model as well as the Ambartsumian equation by applying the homotopy analysis transform method (HATM). In 2019, the authors of [3] presented a hybrid numerical scheme based on the HATM in order to detect the fractional model of nonlinear wave-like equations. In [4], the authors dealt with a fractional extension of the Biswas-Milovic (BM) model by using the fractional homotopy analysis transform method (FHATM). In 2019, Singh et al. [5] developed nondifferentiable solutions of extended wave equations. The author of [6] illustrated the rumor spreading dynamical model involving the Atangana-Baleanu derivative of non-integer order. In addition, fractional differential equations play an important role in modeling mechanics, electrical properties of real materials, rheological theory, aerodynamics, finance, bioengineering and so on. Currently, the fractional calculus and the theory of fractional differential equations have become a popular gambit. Kumar et al. [7] analyzed the exothermic reactions model by using fractional energy balance equation (FEBE). Making use of the CaputoFabrizio fractional operator and the fixed point theorem, the authors [8] reported a fractional SIRS-SI model. See Refs. [9-12].

The research of the oscillation of the fractional differential equations has been done more and more extensively. Zhou et al. [13, 14] suggested the sufficient conditions for the existence of nonoscillatory solutions for fractional neutral differential equations. In 2019, the authors [15] utilized the sufficient criteria for the oscillation of all solutions to the fractional partial differential equation. However, to the best of the author's knowledge very little is known about the oscillation criteria of the fractional partial differential equations involved with the Riemann-Liouville fractional partial differential up to now [16-23].

(c) The Author(s) 2019. This article is distributed under the terms of the Creative Commons Attribution 4.0 International License (http://creativecommons.org/licenses/by/4.0/), which permits unrestricted use, distribution, and reproduction in any medium, provided you give appropriate credit to the original author(s) and the source, provide a link to the Creative Commons license, and indicate if changes were made. 
A lot of work of the fractional differential equations which raise more and more attention has been published lately [24]. At the same time, several novel methods have been established to verify the sufficient condition of the oscillation properties of the fractional partial differential equations, such as [25-33]. We study the sufficient condition for oscillation of the solutions by using the generalized Riccati substitution, fractional integral as well as the properties of the Riemann-Liouville fractional derivative. In order to illustrate the main results, we give several examples at the end of the paper.

The Riemann-Liouville fractional derivative may always be used to solve the oscillation of fractional partial differential equations. Li [34] investigated the forced oscillation of fractional partial differential equations of the form,

$$
\begin{gathered}
D_{+, t}^{\alpha} u(x, t)=a(t) \Delta u(x, t)-m(x, t, u(x, t))+f(x, t), \\
(x, t) \in \Omega \times R_{+} \equiv G .
\end{gathered}
$$

Prakash et al. [35] investigated the oscillation of certain nonlinear fractional partial differential equation with damping term,

$$
\begin{aligned}
& D_{+, t}^{\alpha}\left(r(t) D_{+, t}^{\alpha} u(x, t)\right)+p(t) D_{+, t}^{\alpha} u(x, t)+q(x, t) f(u(x, t)) \\
& \quad=a(t) \Delta u(x, t)+g(x, t), \quad(x, t) \in G .
\end{aligned}
$$

Harikrishnan et al. [36] established the oscillation of the fractional differential equation of the form,

$$
\begin{aligned}
& D_{+, t}^{\alpha}\left(r(t) D_{+, t}^{\alpha} u(x, t)\right)+q(x, t) f(u(x, t)) \\
& \quad=a(t) \Delta u(x, t)+g(x, t), \quad(x, t) \in G .
\end{aligned}
$$

In [37], Wang and Meng studied the oscillatory behavior of a fractional partial differential equation of this form,

$$
\begin{aligned}
D_{+, t}^{\alpha}\left(r(t) D_{+, t}^{\alpha} u(x, t)\right)+p(t) D_{+, t}^{\alpha} u(x, t) & \\
+ & q(x, t) f\left(\int_{0}^{t}(t-v)^{-\alpha} u(x, v) d v\right) \\
= & a(t) \Delta u(x, t), \quad(x, t) \in \Omega \times R_{+} \equiv G .
\end{aligned}
$$

In this paper, we shall investigate the oscillation criteria for the fractional partial differential equation

$$
\begin{aligned}
& D_{+, t}^{\alpha}\left(r(t) D_{+, t}^{\alpha} u(x, t)\right)+p(t) D_{+, t}^{\alpha} u(x, t)+q(x, t) f(u(x, t)) \\
& \quad=a(t) \Delta u(x, t)+\sum_{i=1}^{m} b_{i}(t) \Delta u\left(x, t-\tau_{i}\right), \quad(x, t) \in \Omega \times R_{+} \equiv G,
\end{aligned}
$$

with the Robin boundary condition

$$
\frac{\partial u(x, t)}{\partial N}+g(x, t) u(x, t)=0, \quad(x, t) \in \partial \Omega \times R_{+},
$$


where $\alpha \in(0,1)$ is a constant, $D_{+, t}^{\alpha}$ is the Riemann-Liouville fractional derivative of order $\alpha$ of $u$ with respect $t, \Omega$ is a bounded domain in $R^{n}$ with piecewise smooth boundary $\partial \Omega$, $\Delta$ is the Laplacian operator and $N$ is the unit exterior normal vector to $\partial \Omega$, and $g(x, t)$ is a nonnegative continuous function on $\partial \Omega \times R_{+}$.

Throughout, we assume that:

$\left(A_{1}\right) r(t) \in C^{\alpha}\left(R_{+}, R_{+}\right), p(t) \in C\left(R_{+}, R\right), a(t) \in C\left(R_{+}, R_{+}\right), b_{i}(t) \in C\left(R_{+}, R_{+}\right), \tau_{i} \geq 0$ is a constant, $i=1,2, \ldots, m$;

$\left(A_{2}\right) q(x, t) \in C\left(\bar{G}, R_{+}\right), \min _{x \in \Omega} q(x, t)=Q(t)$;

$\left(A_{3}\right) f: R \rightarrow R$ is a continuous function such that $\frac{f(x)}{x} \geq m$ for certain constant $m>0$ and for all $x \neq 0$.

A solution $u(x, t)$ of (5) is called oscillatory in $G$ if it is neither eventually positive nor eventually negative. Otherwise, it is called nonoscillatory.

\section{Preliminaries and basic lemmas}

In this section, we list several symbols and lemmas which are useful through this paper.

Definition 2.1 ([25]) The Riemann-Liouville fractional integral $I_{+}^{\alpha} y$ of order $\alpha \in R_{+}$is defined by

$$
\left(I_{+}^{\alpha} y\right)(t)=\frac{1}{\Gamma(\alpha)} \int_{0}^{t}(t-v)^{\alpha-1} y(v) d v, \quad t>0,
$$

where $\Gamma(\alpha)$ is the gamma function defined by $\Gamma(\alpha)=\int_{0}^{+\infty} s^{\alpha-1} e^{-s} d s$ for $\alpha>0$. This integral is called left-sided fractional integral.

Definition 2.2 ([25]) The Riemann-Liouville fractional partial derivative of order $0<\alpha<$ 1 of a function $u(x, t)$ is defined by

$$
\left(D_{+, t}^{\alpha} u\right)(x, t)=\frac{\partial}{\partial t} \frac{1}{\Gamma(1-\alpha)} \int_{0}^{t}(t-v)^{-\alpha} u(x, v) d v, \quad t>0,
$$

provided the right hand side is pointwise defined on $R_{+}$, where $\Gamma$ is the gamma function.

Definition 2.3 ([25]) The Riemann-Liouville fractional derivative of order $\alpha>0$ of a function $y: R_{+} \rightarrow R$ on the half-axis $R_{+}$is given by

$$
\begin{aligned}
\left(D_{+}^{\alpha} y\right)(t) & =\frac{d^{\lceil\alpha\rceil}}{d t^{\lceil\alpha\rceil}}\left(I_{+}^{\lceil\alpha\rceil-\alpha} y\right)(t) \\
& =\frac{1}{\Gamma(\lceil\alpha\rceil-\alpha)} \frac{d^{\lceil\alpha\rceil}}{d t^{\lceil\alpha\rceil}} \int_{0}^{t}(t-v)^{\lceil\alpha\rceil-\alpha-1} y(v) d v, \quad t>0,
\end{aligned}
$$

provided the right hand side is pointwise defined on $R_{+}$, where $\lceil\alpha\rceil$ is the ceiling function of $\alpha$.

Lemma 2.1 (Lemma 2.4, [30]) Let

$$
F(t)=\int_{0}^{t}(t-v)^{-\alpha} y(v) d v, \quad \alpha \in(0,1), t>0
$$


Then

$$
F^{\prime}(t)=\Gamma(1-\alpha)\left(D_{+}^{\alpha} y\right)(t)
$$

Lemma 2.2 ([25]) Let $\alpha \geq 0, m \in N$, and $D=\frac{d}{d x}$, If the fractional derivatives $D_{+}^{\alpha} y(t)$ and $D_{+}^{\alpha+m} y(t)$ exist, then

$$
D^{m}\left(D_{+}^{\alpha} y(t)\right)=D_{+}^{\alpha+m} y(t) .
$$

Lemma 2.3 ([25]) Let $\alpha \in(0,1)$ and $I_{+}^{1-\alpha} y(t)$ be the fractional integral (7) of order $1-\alpha$, then

$$
I_{+}^{\alpha}\left(D_{+}^{\alpha} y(t)\right)=y(t)-\frac{I_{+}^{1-\alpha} y(0)}{\Gamma(\alpha)} t^{\alpha-1} .
$$

For convenience, we use the following notations in this paper:

$$
\begin{aligned}
& v(t)=\int_{\Omega} u(x, t) d x, \quad \xi=\frac{t^{\alpha}}{\Gamma(1+\alpha)}, \quad \tilde{c}(\xi)=c(t), \quad \tilde{r}(\xi)=r(t), \\
& \tilde{\sigma}(\xi)=\sigma(t), \quad \xi_{0}=\frac{t_{0}^{\alpha}}{\Gamma(1+\alpha)}, \quad \xi_{1}=\frac{t_{1}^{\alpha}}{\Gamma(1+\alpha)}, \quad \tilde{Q}(\xi)=Q(t), \\
& R(t)=I_{+}^{\alpha}\left(\frac{p(t)}{r(t)}\right) .
\end{aligned}
$$

\section{Main results}

Theorem 3.1 Let condition $\left(A_{1}\right)-\left(A_{3}\right)$ hold, suppose that there exists a function $\varphi \in$ $C^{1}\left[\left[t_{0}, \infty\right),(0, \infty)\right]$, such that

$$
\begin{aligned}
& \int_{t_{0}}^{\infty} \frac{1}{r(s) e^{R(s)}} d s=\infty, \\
& \limsup _{t \rightarrow \infty} A(t)>0, \quad \liminf _{t \rightarrow \infty} A(t)<0,
\end{aligned}
$$

where

$$
A(t)=\frac{1}{\Gamma(\alpha)} \int_{0}^{t}(t-\xi)^{\alpha-1}\left(-m e^{R(\xi)} \varphi(\xi) Q(\xi)+\frac{\left(D_{+}^{\alpha} \varphi(\xi)\right)^{2} e^{R(\xi)} r(\xi)}{4 \varphi(\xi)}\right) d \xi .
$$

Then every solution of (5) is oscillatory.

Proof Suppose to the contrary that $u$ is a nonoscillatory solution of (5). Without loss of generality, we can assume that there exists $u(x, t)>0$ and $u\left(x, t-\tau_{i}\right)>0$ in $G \times\left[t_{0}, \infty\right)$ for some $t_{0}>0, i=1,2, \ldots, m$. Integrating (5) with respect to $x$ over the domain $\Omega$, we obtain

$$
\begin{aligned}
& \int_{\Omega} D_{+, t}^{\alpha}\left(r(t) D_{+, t}^{\alpha} u(x, t)\right) d x \\
& \quad+p(t) \int_{\Omega} D_{+, t}^{\alpha} u(x, t) d x+\int_{\Omega} q(x, t) f(u(x, t)) d x \\
& =a(t) \int_{\Omega} \Delta u(x, t) d x+\int_{\Omega} \sum_{i=1}^{m} b_{i}(t) \Delta u\left(x, t-\tau_{i}\right) d x .
\end{aligned}
$$


Using Green's formula, it is obvious that

$$
\begin{aligned}
\int_{\Omega} \Delta u(x, t) d x= & \int_{\partial \Omega} \frac{\partial u(x, t)}{\partial N} d s=-\int_{\partial \Omega} g(x, t) u(x, t) d s \leq 0, \quad t \geq t_{1} \\
\int_{\Omega} \Delta u\left(x, t-\tau_{i}\right) d x & =\int_{\partial \Omega} \frac{\partial u\left(x, t-\tau_{i}\right)}{\partial N} d s \\
& =-\int_{\partial \Omega} g\left(x, t-\tau_{i}\right) u\left(x, t-\tau_{i}\right) d s \\
& \leq 0, \quad t \geq t_{1}, i=1,2, \ldots, m
\end{aligned}
$$

where $d s$ is a surface element on $\partial \Omega$. By using Jensen's inequality and $\left(A_{2}\right)$, we obtain

$$
\int_{\Omega} q(x, t) f(u(x, t)) d x \geq Q(t) f\left(\int_{\Omega} u(x, t) d x\right)=Q(t) f(v(t)) .
$$

Combining (17)-(20), we have

$$
\begin{aligned}
D_{+}^{\alpha}\left(r(t) D_{+}^{\alpha} v(t)\right)+p(t) D_{+}^{\alpha} v(t)+Q(t) f(v(t)) \leq 0, \\
\begin{aligned}
D_{+}^{\alpha}\left(e^{R(t)} r(t) D_{+}^{\alpha} v(t)\right) & =e^{R(t)} \frac{p(t)}{r(t)} r(t) D_{+}^{\alpha} v(t)+D_{+}^{\alpha}\left(r(t) D_{+}^{\alpha} v(t)\right) e^{R(t)} \\
& =e^{R(t)} p(t) D_{+}^{\alpha} v(t)+D_{+}^{\alpha}\left(r(t) D_{+}^{\alpha} v(t)\right) e^{R(t)} \\
& \leq e^{R(t)}(-Q(t) f(v(t))) \\
& <0 .
\end{aligned}
\end{aligned}
$$

Then $e^{R(t)} r(t) D_{+}^{\alpha} v(t)$ is strictly decreasing on $\left[t_{1}, \infty\right)$, and thus $D_{+}^{\alpha} v(t)$ is eventually of one sign. We claim $D_{+}^{\alpha} v(t) \geq 0$ on $\left[t_{2}, \infty\right)$, where $t_{2}>t_{1}$ is sufficiently large. Otherwise, assume there exists a sufficiently large $T>t_{2}$ such that $D_{+}^{\alpha} v(t)<0$ on $[T, \infty)$. Then, for $t \in[T, \infty)$, by Lemma 2.1, we have

$$
\frac{F^{\prime}(t)}{\Gamma(1-\alpha)}=D_{+}^{\alpha} v(t) \leq \frac{r(T) e^{R(T)}\left(D_{+}^{\alpha} \nu(T)\right)}{r(t) e^{R(t)}}
$$

where

$$
F(t)=\int_{0}^{t}(t-s)^{-\alpha} v(s) d s
$$

Integrating the above inequality from $T$ to $t$, we have

$$
F(t) \leq F(T)+\Gamma(1-\alpha) r(T) e^{R(T)}\left(D_{+}^{\alpha} \nu(T)\right) \int_{T}^{t} \frac{1}{r(s) e^{R(s)}} d s .
$$

Letting $t \rightarrow \infty$, we get $\lim _{t \rightarrow \infty} F(t) \leq-\infty$ which is a contradiction. Hence $D_{+}^{\alpha} v(t) \geq 0$ for $t \geq t_{1}$ holds. Define the function $\omega$ by the generalized Riccati substitution

$$
\omega(t)=\varphi(t) \frac{e^{R(t)} r(t) D_{+}^{\alpha} v(t)}{v(t)}, \quad t \geq t_{1}
$$




$$
\begin{aligned}
D_{+}^{\alpha} \omega(t)= & \left(D_{+}^{\alpha} \varphi(t)\right) \frac{\omega(t)}{\varphi(t)}+D_{+}^{\alpha}\left(\frac{e^{R(t)} r(t) D_{+}^{\alpha} v(t)}{v(t)}\right) \varphi(t) \\
= & \left(D_{+}^{\alpha} \varphi(t)\right) \frac{\omega(t)}{\varphi(t)}+D_{+}^{\alpha}\left(e^{R(t)} r(t) D_{+}^{\alpha} v(t)\right) \frac{1}{v(t)} \varphi(t) \\
& +D_{+}^{\alpha}\left(\frac{1}{v(t)}\right) e^{R(t)} r(t) D_{+}^{\alpha} v(t) \varphi(t) \\
\leq & \left(D_{+}^{\alpha} \varphi(t)\right) \frac{\omega(t)}{\varphi(t)}+\frac{\varphi(t) e^{R(t)}(-Q(t) f(v(t)))}{v(t)} \\
& -\frac{\left(D_{+}^{\alpha} v(t)\right)^{2} e^{R(t)} r(t) \varphi(t)}{v^{2}(t)} \\
\leq & \left(D_{+}^{\alpha} \varphi(t)\right) \frac{\omega(t)}{\varphi(t)}+\frac{\varphi(t) e^{R(t)}(-Q(t) m v(t))}{v(t)}-\frac{\omega^{2}(t)}{\varphi(t) e^{R(t)} r(t)} \\
= & -m e^{R(t)} \varphi(t) Q(t)-\left(\frac{\omega(t)}{\sqrt{\varphi(t) e^{R(t)} r(t)}}-\frac{D_{+}^{\alpha} \varphi(t) \sqrt{e^{R(t)} r(t)}}{2 \sqrt{\varphi(t)}}\right)^{2} \\
& +\frac{\left(D_{+}^{\alpha} \varphi(t)\right)^{2} e^{R(t)} r(t)}{4 \varphi(t)} \\
\leq & -m e^{R(t)} \varphi(t) Q(t)+\frac{\left(D_{+}^{\alpha} \varphi(t)\right)^{2} e^{R(t)} r(t)}{4 \varphi(t)} .
\end{aligned}
$$

By Lemma 2.3, we have

$$
I_{+}^{\alpha}\left(D_{+}^{\alpha} \omega(t)\right)=\omega(t)-\frac{I_{+}^{1-\alpha} \omega(0)}{\Gamma(\alpha)} t^{\alpha-1} .
$$

According to (26), we have

$$
I_{+}^{\alpha}\left(D_{+}^{\alpha} \omega(t)\right) \leq I_{+}^{\alpha}\left(-m e^{R(t)} \varphi(t) Q(t)+\frac{\left(D_{+}^{\alpha} \varphi(t)\right)^{2} e^{R(t)} r(t)}{4 \varphi(t)}\right) .
$$

Then we have

$$
\omega(t)-\frac{I_{+}^{1-\alpha} \omega(0)}{\Gamma(\alpha)} t^{\alpha-1} \leq I_{+}^{\alpha}\left(-m e^{R(t)} \varphi(t) Q(t)+\frac{\left(D_{+}^{\alpha} \varphi(t)\right)^{2} e^{R(t)} r(t)}{4 \varphi(t)}\right) .
$$

Let $B=\frac{I_{+}^{1-\alpha} \omega(0)}{\Gamma(\alpha)}$, then

$$
\begin{aligned}
\omega(t) & \leq I_{+}^{\alpha}\left(-m e^{R(t)} \varphi(t) Q(t)+\frac{\left(D_{+}^{\alpha} \varphi(t)\right)^{2} e^{R(t)} r(t)}{4 \varphi(t)}\right)+\frac{I_{+}^{1-\alpha} \omega(0)}{\Gamma(\alpha)} t^{\alpha-1} \\
& =A(t)+B t^{\alpha-1} .
\end{aligned}
$$

Letting $t \rightarrow \infty$ in (30), we have

$$
\liminf _{t \rightarrow \infty} \omega(t) \leq \liminf _{t \rightarrow \infty} A(t)+\limsup _{t \rightarrow \infty} B t^{\alpha-1}<0
$$

which contradicts $\omega(t) \geq 0$, similarly, if $u(x, t)<0$ we can get the contradiction. The proof is complete. 
For the following theorem, we introduce a class of functions $R$. Let

$$
D=\left\{(t, s): t \geq s \geq t_{0}\right\}
$$

The function $H \in C(D, R)$ is said to belong to the class $R$, if

(i) $H(t, t)=0$, for $t \geq t_{0}, H(t, s)>0$, for $t \neq s$;

(ii) $H(t, s)$ has partial derivatives on $D$ such that $\frac{\partial H}{\partial t}(t, s)=h_{1}(t, s) \sqrt{H(t, s)}$, $\frac{\partial H}{\partial s}(t, s)=-h_{2}(t, s) \sqrt{H(t, s)}$, for some $h_{1}, h_{2} \in L_{\mathrm{loc}}^{1}(D, R)$.

Theorem 3.2 Suppose conditions $\left(A_{1}\right)-\left(A_{3}\right)$ hold, and (14)-(15) are also true. If, for any $T \geq t_{0}$, there exists an interval $(a, b) \in[T, \infty)$, and there exists $a c \in(a, b), H \in R$, such that

$$
\begin{aligned}
& \frac{1}{H(c, a)}\left\{\int_{a}^{c} e^{\tilde{R}(s)} \tilde{\varphi}(s) \tilde{Q}(s) m H(s, a) d s-\int_{a}^{c} \frac{\tilde{\varphi}(s) e^{\tilde{R}(s)} \tilde{r}(s)}{4}\right. \\
& \left.\quad \times\left(h_{1}(s, a)+\frac{\tilde{\varphi}^{\prime}(s) \sqrt{H(s, a)}}{\tilde{\varphi}(s)}\right)^{2} d s\right\}+\frac{1}{H(b, c)}\left\{\int_{c}^{b} e^{\tilde{R}(s)} \tilde{\varphi}(s) \tilde{Q}(s) m\right. \\
& \left.\quad \times H(b, s) d s-\int_{c}^{b} \frac{\tilde{\varphi}(s) e^{\tilde{R}(s)} \tilde{r}(s)}{4}\left(-h_{2}(b, s)+\frac{\tilde{\varphi}^{\prime}(s) \sqrt{H(b, s)}}{\tilde{\varphi}(s)}\right)^{2} d s\right\}
\end{aligned}
$$

$>0$,

then every solution of (5) is oscillatory.

Proof Suppose to the contrary that $u(x, t)$ is a nonoscillatory solution of (5). Without loss of generality, we can assume that there exists $u(x, t)>0$ and $u\left(x, t-\tau_{i}\right)>0$ in $G \times\left[t_{0}, \infty\right)$ for some $t_{0}>0, i=1,2, \ldots, m$. Proceeding as in the proof of Theorem 3.1, according to (26), we have

$$
D_{+}^{\alpha} \omega(t) \leq\left(D_{+}^{\alpha} \varphi(t)\right) \frac{\omega(t)}{\varphi(t)}-m e^{R(t)} \varphi(t) Q(t)-\frac{\omega^{2}(t)}{\varphi(t) e^{R(t)} r(t)} .
$$

Let $\omega(t)=\tilde{\omega}(\xi)$. Then $D_{+}^{\alpha} \omega(t)=\tilde{\omega}^{\prime}(\xi)$ and $D_{+}^{\alpha} \varphi(t)=\tilde{\varphi}^{\prime}(\xi)$. So the above inequality is transformed into

$$
\tilde{\omega}^{\prime}(\xi) \leq \tilde{\varphi}^{\prime}(\xi) \frac{\tilde{\omega}(\xi)}{\tilde{\varphi}(\xi)}-m e^{\tilde{R}(\xi)} \tilde{\varphi}(\xi) \tilde{Q}(\xi)-\frac{\tilde{\omega}^{2}(\xi)}{\tilde{\varphi}(\xi) e^{\tilde{R}(\xi)} \tilde{r}(\xi)}
$$

Multiplying both sides of (35) by $H(s, t)$, integrating from $t$ to $c$ about $s$, where $t \in(a, c]$,

$$
\begin{aligned}
& \int_{t}^{c} \tilde{\omega}^{\prime}(s) H(s, t) d s=\tilde{\omega}(c) H(c, t)-\int_{t}^{c} h_{1}(s, t) \sqrt{H(s, t)} \tilde{\omega}(s) d s, \\
& \int_{t}^{c} e^{\tilde{R}(s)} \tilde{\varphi}(s) \tilde{Q}(s) m H(s, t) d s \\
& \quad \leq-\tilde{\omega}(c) H(c, t)+\int_{t}^{c} h_{1}(s, t) \sqrt{H(s, t)} \tilde{\omega}(s) d s+\int_{t}^{c} H(s, t) \tilde{\varphi}^{\prime}(s) \frac{\tilde{\omega}(s)}{\tilde{\varphi}(s)} d s \\
& \quad-\int_{t}^{c} \frac{\tilde{\omega}^{2}(s)}{\tilde{\varphi}(s) e^{\tilde{R}(s)} \tilde{r}(s)} H(s, t) d s
\end{aligned}
$$




$$
\begin{aligned}
= & -\tilde{\omega}(c) H(c, t)-\int_{t}^{c}\left\{\frac{\tilde{\omega}(s) \sqrt{H(s, t)}}{\sqrt{\tilde{\varphi}(s) e^{\tilde{R}(s)} \tilde{r}(s)}}\right. \\
& \left.-\frac{\frac{\tilde{\varphi}^{\prime}(s) H(s, t) \tilde{\omega}(s)}{\tilde{\varphi}(s)}+h_{1}(s, t) \sqrt{H(s, t)} \tilde{\omega}(s)}{2 \tilde{\omega}(s) \sqrt{H(s, t)}} \sqrt{\tilde{\varphi}(s) e^{\tilde{R}(s)} \tilde{r}(s)}\right\}^{2} d s \\
& +\int_{t}^{c}\left(\frac{\frac{\tilde{\varphi}^{\prime}(s) H(s, t) \tilde{\omega}(s)}{\tilde{\varphi}(s)}+h_{1}(s, t) \sqrt{H(s, t)} \tilde{\omega}(s)}{2 \tilde{\omega}(s) \sqrt{H(s, t)}}\right)^{2} \tilde{\varphi}(s) e^{\tilde{R}(s)} \tilde{r}(s) d s \\
\leq & -\tilde{\omega}(c) H(c, t) \\
& +\int_{t}^{c}\left(\frac{\frac{\tilde{\varphi}^{\prime}(s) \sqrt{H(s, t)}}{\tilde{\varphi}(s)}+h_{1}(s, t)}{2}\right)^{2} \tilde{\varphi}(s) e^{\tilde{R}(s)} \tilde{r}(s) d s .
\end{aligned}
$$

Multiplying both sides of (35) by $H(t, s)$, integrating from $c$ to $t$ over $s$, where $t \in[c, b)$,

$$
\begin{aligned}
& \int_{c}^{t} \tilde{\omega}^{\prime}(s) H(t, s) d s=-\tilde{\omega}(c) H(t, c)+\int_{c}^{t} \sqrt{H(t, s)} h_{2}(t, s) \tilde{\omega}(s) d s, \\
& \int_{c}^{t} e^{\tilde{R}(s)} \tilde{\varphi}(s) \tilde{Q}(s) m H(t, s) d s \\
& \leq \tilde{\omega}(c) H(t, c)-\int_{c}^{t} \sqrt{H(t, s)} h_{2}(t, s) \tilde{\omega}(s) d s+\int_{c}^{t} H(t, s) \tilde{\varphi}^{\prime}(s) \frac{\tilde{\omega}(s)}{\tilde{\varphi}(s)} d s \\
& -\int_{c}^{t} \frac{\tilde{\omega}^{2}(s)}{\tilde{\varphi}(s) e^{\tilde{R}(s)} \tilde{r}(s)} H(t, s) d s \\
& =\tilde{\omega}(c) H(t, c)-\int_{c}^{t}\left\{\frac{\tilde{\omega}(s) \sqrt{H(t, s)}}{\sqrt{\tilde{\varphi}(s) e^{\tilde{R}(s)} \tilde{r}(s)}}\right. \\
& \left.-\frac{\frac{\tilde{\varphi}^{\prime}(s) H(t, s) \tilde{\omega}(s)}{\tilde{\varphi}(s)}-h_{2}(t, s) \sqrt{H(t, s)} \tilde{\omega}(s)}{2 \tilde{\omega}(s) \sqrt{H(t, s)}} \sqrt{\tilde{\varphi}(s) e^{\tilde{R}(s)} \tilde{r}(s)}\right\}^{2} d s \\
& +\int_{c}^{t}\left(\frac{\frac{\tilde{\varphi}^{\prime}(s) H(t, s) \tilde{\omega}(s)}{\tilde{\varphi}(s)}-h_{2}(t, s) \sqrt{H(t, s)} \tilde{\omega}(s)}{2 \tilde{\omega}(s) \sqrt{H(t, s)}}\right)^{2} \tilde{\varphi}(s) e^{\tilde{R}(s)} \tilde{r}(s) d s \\
& \leq \tilde{\omega}(c) H(t, c) \\
& +\int_{c}^{t}\left(\frac{\frac{\tilde{\varphi}^{\prime}(s) \sqrt{H(t, s)}}{\tilde{\varphi}(s)}-h_{2}(t, s)}{2}\right)^{2} \tilde{\varphi}(s) e^{\tilde{R}(s)} \tilde{r}(s) d s
\end{aligned}
$$

Letting $t \rightarrow a^{+}$in (37) and $t \rightarrow b^{-}$in (39),

$$
\begin{aligned}
& \frac{1}{H(c, a)}\left\{\int_{a}^{c} e^{\tilde{R}(s)} \tilde{\varphi}(s) \tilde{Q}(s) m H(s, a) d s-\int_{a}^{c} \frac{\tilde{\varphi}(s) e^{\tilde{R}(s)} \tilde{r}(s)}{4}\right. \\
& \left.\quad \times\left(h_{1}(s, a)+\frac{\tilde{\varphi}^{\prime}(s) \sqrt{H(s, a)}}{\tilde{\varphi}(s)}\right)^{2} d s\right\}+\frac{1}{H(b, c)}\left\{\int_{c}^{b} e^{\tilde{R}(s)} \tilde{\varphi}(s) \tilde{Q}(s) m\right. \\
& \left.\quad \times H(b, s) d s-\int_{c}^{b} \frac{\tilde{\varphi}(s) e^{\tilde{R}(s)} \tilde{r}(s)}{4}\left(-h_{2}(b, s)+\frac{\tilde{\varphi}^{\prime}(s) \sqrt{H(b, s)}}{\tilde{\varphi}(s)}\right)^{2} d s\right\} \\
& \leq 0
\end{aligned}
$$

which contradicts Eq. (33). The proof is complete. 
Theorem 3.3 Suppose conditions $\left(A_{1}\right)-\left(A_{3}\right)$ hold, and (14)-(15) are also true. If, for any $T \geq t_{0}$, there exists an interval $(a, b) \in[T, \infty)$, and there exists $a c \in(a, b), H \in R$, such that

$$
\begin{aligned}
& \limsup _{t \rightarrow \infty}\left\{\int_{l}^{t} e^{\tilde{R}(s)} \tilde{\varphi}(s) \tilde{Q}(s) m H(s, l) d s-\int_{l}^{t} \frac{\tilde{\varphi}(s) e^{\tilde{R}(s)} \tilde{r}(s)}{4}\right. \\
& \left.\quad \times\left(h_{1}(s, l)+\frac{\tilde{\varphi}^{\prime}(s) \sqrt{H(s, l)}}{\tilde{\varphi}(s)}\right)^{2} d s\right\}>0, \\
& \limsup _{t \rightarrow \infty}\left\{\int_{l}^{t} e^{\tilde{R}(s)} \tilde{\varphi}(s) \tilde{Q}(s) m H(t, s) d s-\int_{l}^{t} \frac{\tilde{\varphi}(s) e^{\tilde{R}(s)} \tilde{r}(s)}{4}\right. \\
& \left.\quad \times\left(-h_{2}(t, s)+\frac{\tilde{\varphi}^{\prime}(s) \sqrt{H(t, s)}}{\tilde{\varphi}(s)}\right)^{2} d s\right\}>0,
\end{aligned}
$$

then, for $l \in\left[t_{0}, \infty\right), t_{1}>t_{0}$ positive, every solution of (5) is oscillatory.

Proof Suppose to the contrary that $u(x, t)$ is a nonoscillatory solution of (5). Without loss of generality, we can assume that there exists $u(x, t)>0$ and $u\left(x, t-\tau_{i}\right)>0$ in $G \times\left[t_{0}, \infty\right)$ for some $t_{2} \geq t_{1}, i=1,2, \ldots, m$.

Letting $l=a \geq t_{2}$ in (41), according to (41) we have $c>a$ such that

$$
\begin{gathered}
\int_{a}^{c} e^{\tilde{R}(s)} \tilde{\varphi}(s) \tilde{Q}(s) m H(s, a) d s-\int_{a}^{c} \frac{\tilde{\varphi}(s) e^{\tilde{R}(s)} \tilde{r}(s)}{4} \\
\times\left(h_{1}(s, a)+\frac{\tilde{\varphi}^{\prime}(s) \sqrt{H(s, a)}}{\tilde{\varphi}(s)}\right)^{2} d s>0 .
\end{gathered}
$$

Letting $l=c \geq t_{2}$ in (42), according to (42) we have $b>c$ such that

$$
\begin{gathered}
\int_{c}^{b} e^{\tilde{R}(s)} \tilde{\varphi}(s) \tilde{Q}(s) m H(b, s) d s-\int_{c}^{b} \frac{\tilde{\varphi}(s) e^{\tilde{R}(s)} \tilde{r}(s)}{4} \\
\quad \times\left(-h_{2}(b, s)+\frac{\tilde{\varphi}^{\prime}(s) \sqrt{H(b, s)}}{\tilde{\varphi}(s)}\right)^{2} d s>0 .
\end{gathered}
$$

According to (43) and (44) we find that (33) is true. Then every solution of (5) is oscillatory.

If we let $H(t, s)=(t-s)^{\lambda}$, where $t \geq s \geq t_{0}$, we have the following lemma.

Lemma 3.1 Suppose conditions $\left(A_{1}\right)-\left(A_{3}\right)$ hold, and (14)-(15) are also true. Then the following inequalities are true:

$$
\begin{aligned}
& \limsup _{t \rightarrow \infty}\left\{\int_{l}^{t} e^{\tilde{R}(s)} \tilde{\varphi}(s) \tilde{Q}(s) m(s-l)^{\lambda} d s-\int_{l}^{t} \frac{\tilde{\varphi}(s) e^{\tilde{R}(s)} \tilde{r}(s)}{4}\right. \\
& \left.\quad \times\left(\lambda(s-l)^{\frac{\lambda-2}{2}}+\frac{\tilde{\varphi}^{\prime}(s)(s-l)^{\frac{\lambda}{2}}}{\tilde{\varphi}(s)}\right)^{2} d s\right\}>0, \\
& \limsup _{t \rightarrow \infty}\left\{\int_{l}^{t} e^{\tilde{R}(s)} \tilde{\varphi}(s) \tilde{Q}(s) m(t-s)^{\lambda} d s-\int_{l}^{t} \frac{\tilde{\varphi}(s) e^{\tilde{R}(s)} \tilde{r}(s)}{4}\right.
\end{aligned}
$$




$$
\left.\times\left(-\lambda(t-s)^{\frac{\lambda-2}{2}}+\frac{\tilde{\varphi}^{\prime}(s)(t-s)^{\frac{\lambda}{2}}}{\tilde{\varphi}(s)}\right)^{2} d s\right\}>0 .
$$

Then, for every $l \geq t_{0}$,Eq. (5) is oscillatory.

In general, considering $H(t, s)=(K(t)-K(s))^{\lambda}$, where $K(t)=\int_{t_{1}}^{t} \frac{1}{r(s)} d s$, we have the following lemma.

Lemma 3.2 Suppose conditions $\left(A_{1}\right)-\left(A_{3}\right)$ hold, and (14)-(15) are also true. Then the following inequalities are true:

$$
\begin{gathered}
\limsup _{t \rightarrow \infty}\left\{\int_{l}^{t} e^{\tilde{R}(s)} \tilde{\varphi}(s) \tilde{Q}(s) m(K(s)-K(l))^{\lambda} d s-\int_{l}^{t} \frac{\tilde{\varphi}(s) e^{\tilde{R}(s)} \tilde{r}(s)}{4}\right. \\
\left.\quad \times\left(\frac{\lambda(K(s)-K(l))^{\frac{\lambda-2}{2}}}{r(s)}+\frac{\tilde{\varphi}^{\prime}(s)(K(s)-K(l))^{\frac{\lambda}{2}}}{\tilde{\varphi}(s)}\right)^{2} d s\right\}>0, \\
\limsup _{t \rightarrow \infty}\left\{\int_{l}^{t} e^{\tilde{R}(s)} \tilde{\varphi}(s) \tilde{Q}(s) m(K(t)-K(s))^{\lambda} d s-\int_{l}^{t} \frac{\tilde{\varphi}(s) e^{\tilde{R}(s)} \tilde{r}(s)}{4}\right. \\
\left.\quad \times\left(-\frac{\lambda(K(t)-K(s))^{\frac{\lambda-2}{2}}}{r(s)}+\frac{\tilde{\varphi}^{\prime}(s)(K(t)-K(s))^{\frac{\lambda}{2}}}{\tilde{\varphi}(s)}\right)^{2} d s\right\}>0 .
\end{gathered}
$$

Then, for every $l \geq t_{0}$, Eq. (5) is oscillatory.

\section{Examples}

Example 4.1 Consider the following fractional partial differential equation:

$$
\begin{gathered}
D_{+, t}^{\alpha}\left(e^{t} D_{+, t}^{\alpha} u(x, t)\right)+(t-1) D_{+, t}^{\alpha} u(x, t)+\left(t^{2}+x^{2}\right)(u(x, t)+1) \\
=t \Delta u(x, t)+5 t \Delta u(x, t-1), \quad(x, t) \in(0, \pi) \times(0, \infty),
\end{gathered}
$$

with the Robin boundary condition

$$
u_{x}(0, t)=u_{x}(\pi, t)=0 .
$$

Notice $\alpha \in(0,1), r(t)=e^{t}, p(t)=t-1, q(x, t)=t^{2}+x^{2}, Q(t)=t^{2}, f(x)=x+1, m=1, a(t)=t$, $b_{1}(t)=5 t, \tau_{1}=1, \Omega=(0, \pi)$.

Then (49) is oscillatory by Theorem 3.1.

Example 4.2 Consider the following fractional partial differential equation:

$$
\begin{aligned}
& D_{+, t}^{\frac{1}{2}}\left(\frac{1}{t} D_{+, t}^{\frac{1}{2}} u(x, t)\right)+t\left(t^{2}-1\right) D_{+, t}^{\frac{1}{2}} u(x, t)+t e^{x} 2 u(x, t) \\
& \quad=e^{t} \Delta u(x, t)+e^{t} \Delta u(x, t-3), \quad(x, t) \in(0, \pi) \times(0, \infty),
\end{aligned}
$$

with the Robin boundary condition

$$
u_{x}(0, t)=u_{x}(\pi, t)=0 .
$$


Notice $\alpha=\frac{1}{2}, r(t)=\frac{1}{t}, p(t)=t\left(t^{2}-1\right), q(x, t)=t e^{x}, Q(t)=t, f(x)=2 x, m=1, a(t)=e^{t}$, $b_{1}(t)=e^{t}, \tau_{1}=3, \Omega=(0, \pi)$.

Then (51) is oscillatory by Theorem 3.1.

\section{Conclusion}

In this paper, we illustrate the sufficient conditions for oscillation criteria of certain fractional partial differential equations by using the generalized Riccati transformation and Riemann-Liouville derivative. The proof has become concise with the aid of fractional calculus and fractional derivatives. The results provide some new methods to research the oscillation criteria of fractional partial differential equations.

\section{Acknowledgements}

The authors are grateful to the reviewers for their valuable and insightful comments.

\section{Funding}

This research is supported by National Natural Science Foundation of China (No. 11671227).

\section{Competing interests}

The authors declare that they have no competing interests.

\section{Authors' contributions}

The authors state that assignments have been finished, respectively. All authors read and approved the final manuscript.

\section{Publisher's Note}

Springer Nature remains neutral with regard to jurisdictional claims in published maps and institutional affiliations.

Received: 29 April 2019 Accepted: 23 October 2019 Published online: 04 November 2019

References

1. Kumar, D., Singh, J., Baleanu, D., Rathore, S.: Analysis of a fractional model Ambartsumian equation. Eur. Phys. J. Plus $133,159(2018)$

2. Singh, J., Kumar, D., Baleanu, D.: On the analysis of fractional diabetes model with exponential law. Adv. Differ. Equ. 2018, 231 (2018)

3. Kumar, D., Singh, J., Purohit, S.D., Swroop, R.: A hybrid analytical algorithm for nonlinear fractional wave-like equations. Math. Model. Nat. Phenom. 14, 304 (2019)

4. Singh, J., Kumar, D., Baleanu, D.: New aspects of fractional Biswas-Milovic model with Mittag-Leffler law. Math. Model. Nat. Phenom. 14(3), 303 (2019)

5. Singh, J., Kumar, D., Baleanu, D., Rathore, S.: On the local fractional wave equation in fractal strings. Math. Methods Appl. Sci. 42(5), 1588-1595 (2019)

6. Singh, J.: A new analysis for fractional rumor spreading dynamical model in a social network with Mittag-Leffler law. Chaos 29, 013137 (2019)

7. Kumar, D., Singh, J., Tanwar, K., Baleanu, D.: A new fractional exothermic reactions model having constant heat source in porous media with power, exponential and Mittag-Leffler laws. Int. J. Heat Mass Transf. 138, 1222-1227 (2019)

8. Kumar, D., Singh, J., Qurashi, M.A., Baleanu, D.: A new fractional SIRS-SI malaria disease model with application of vaccines, antimalarial drugs, and spraying. Adv. Differ. Equ. 2019, 278 (2019). https://doi.org/10.1186/s13662-019-2199-9

9. Feng, Q.H., Meng, F.W.: Oscillation of solutions to nonlinear forced fractional differential equations. Electron. J. Differ. Equ. 2013, 169 (2013)

10. Prakash, P., Harikrishnan, S., Nieto, J., et al.: Oscillation of a time fractional partial differential equation. Electron. J. Qual. Theory Differ. Equ. 2014, 15 (2014)

11. Li, W.N.: Forced oscillation criteria for a class of fractional partial differential equations with damping term. Math Probl. Eng. 2015, 6 (2015)

12. Meng, F.: An oscillation theorem for second order superlinear differential equations. Indian J. Pure Appl. Math., 27 651-658 (1996)

13. Zhou, Y., Ahmad, B., Alsaedi, A.: Existence of nonoscillatory solutions for fractional neutral differential equations. Appl. Math. Lett. 72, 70-74 (2017)

14. Zhou, Y., Ahmad, B., Alsaedi, A.: Existence of nonoscillatory solutions for fractional functional differential equations. Bull. Malays. Math. Soc. 42(2), 751-766 (2019)

15. Zhou, Y., Ahmad, B., Chen, F., Alsaedi, A.: Oscillation for fractional partial differential equations. Bull. Malays. Math. Soc. 42(2), 449-465 (2019)

16. Wang, J., Meng, F., Liu, S.: Integral average method for oscillation of second order partial differential equations with delays. Appl. Math. Comput. 187, 815-823 (2007)

17. Wang, J., Meng, F., Liu, S.: Interval oscillation criteria for second order partial differential equations with delays. J. Comput. Appl. Math. 212(2), 397-405 (2008) 
18. Lu, F., Meng, F:: Oscillation theorems for superlinear second-order damped differential equations. Appl. Math. Comput. 189(1), 796-804 (2007)

19. Feng, Q., Meng, F.: Oscillation of solutions to nonlinear forced fractional differential equations. Electron. J. Differ. Equ. 2013, 169 (2013)

20. Prakash, P., Harikrishnan, S., Nieto, J.J., Kim, J.H.: Oscillation of a time fractional partial differential equation. Electron. J. Qual. Theory Differ. Equ. (2014). https://doi.org/10.14232/ejatde.2014.1.15

21. Qi, C., Cheng, J.: Interval oscillation criteria for a class of fractional differential equations with damping term. Math. Probl. Eng. 2013, 8 (2013)

22. Grace, S.R., Agarwal, R.P., Wong, P.J.Y., Zafer, A.: On the oscillation of fractional differential equations. Fract. Calc. Appl. Anal. 15(2), 222-231 (2012)

23. Courant, R., Hilbert, D.: Methods of Mathematical Physics. Interscience, New York (1966)

24. Yang, J., Liu, A., Liu, T.: Forced oscillation of nonlinear fractional differential equations with damping term. Adv. Differ Equ. 2015, 7 (2015)

25. Kilbas, A.A., Srivastava, H.M., Trujillo, J.J.: Theory and Applications of Fractional Differential Equations. Elsevier, Amsterdam (2006)

26. Chen, D.X:: Oscillation criteria of fractional differential equations. Adv. Differ. Equ. 2012, 10 (2012)

27. Podlubny, I.: Fractional Differential Equations. Academic Press, San Diego (1999)

28. Zhou, Y: Basic Theory of Fractional Differential Equations. World Science Publishing, Hackensack (2014)

29. Li, W.N., Sheng, W.: Oscillation properties for solutions of a kind of partial fractional differential equations with damping term. J. Nonlinear Sci. Appl. 9(4), 1600-1608 (2016)

30. Chen, D.X., Qu, P.X., Lan, Y.H.: Forced oscillation of certain fractional differential equations. Adv. Differ. Equ. 2013, 125 (2013)

31. Leibniz, G.W.: Mathematische Schriften. Georg Olms Verlags Buchhand, Hildesheim (1962)

32. Kilbas, A.A., Srivastava, H.M., Trujillo, J.J.: Theory and Applications of Fractional Differential Equations. Elsevier, Amsterdam (2006)

33. Samko, S.G., Kilbas, A.A., Marichev, O.: Fractional Integral and Derivatives: Theory and Applications. Elsevier, Amsterdam (1993)

34. Li, W.N.: On the forced oscillation of certain fractional partial differential equations. Appl. Math. Lett. 50, 5-9 (2015)

35. Prakash, P., Harikrishnan, S., Benchohrab, M.: Oscillation of certain nonlinear fractional partial differential equation with damping term. Appl. Math. Lett. 43, 72-79 (2015)

36. Harikrishnan, P., Prakash, J., Nieto, J.: Forced oscillation of solutions of a nonlinear fractional partial differential equation. Appl. Math. Comput. 254, 14-19 (2015)

37. Wang, J., Meng, F:. Oscillatory behavior of a fractional partial differential equation. J. Appl. Anal. Comput. 8, 1011-1020 (2018)

\section{Submit your manuscript to a SpringerOpen ${ }^{0}$ journal and benefit from:}

- Convenient online submission

- Rigorous peer review

- Open access: articles freely available online

- High visibility within the field

- Retaining the copyright to your article

Submit your next manuscript at $\gg$ springeropen.com 